\title{
DISCONTINUOUS PHASE TRANSITIONS IN $S$-PAIRED FERMI SYSTEMS
}

\author{
M. Mulak and R. Gonczarek \\ Institute of Physics, Technical University of Wrocław \\ Wybrzeże Wyspiańskiego 27, 50-370 Wrocław, Poland \\ (Received April 15, 1997; in final form July 14, 1997)
}

\begin{abstract}
The most general $S$-paired systems of fermions for which a gap equation can be separated according to the specific redefined variables, are considered. Moreover, a solution of this gap equation (gap energy) is obtained in a parametric form. It allows us to investigate thermodynamic properties of such systems i.e. the thermodynamic potential, entropy, heat capacity and critical magnetic induction. We show also that the heat capacity difference between the superconducting and the normal phase is entirely determined by the order parameter. The obtained results reveal possibilities of discontinuous phase transition to the normal state and moreover, the theorem on existence of such transitions is formulated and proved. The condition for the BCS-type energy gap is given, which additionally classifies properties of the heat capacity of $S$-paired phase by virtue of the shape of the energy gap. In order to illustrate the developed formalism, the numerical results for the so-called statistical spin liquids and application of the established methods to the experimental data conversion, are presented and discussed.
\end{abstract}

PACS numbers: 74.20.-z

\section{Introduction}

The purpose of the present paper is to formulate the general methods of investigation of $S$-paired systems of fermions. Our considerations constitute the generalization of the results obtained applying Green's functions formalism in the framework of weak coupling approximation [1-3].

Assuming a very plain properties of the gap equation for $S$-paired systems [1], which always occur in a weak coupling formalism, we can admit a comprehensive class of gap equation solutions attainable in various microscopic approaches.

In order to study and qualify the thermodynamic properties of any $S$-paired system, we should consider functions such as thermodynamic potential, entropy and heat capacity. In this paper we show that only the simple assumption on a gap equation structure allows us to find out precise formulas for differences 
of these thermodynamic functions between the superconducting and the normal state. Consequently, the obtained parametric form of the thermodynamic potential difference, between the $S$-paired and normal phase, admits to considerations the discontinuous order parameter i.e. the leap of energy gap to zero at the phase transition point. Such behaviour of the order parameter determines the type of phase transition, which should be type I in the considered case. Moreover, the critical temperature for discontinuous phase transition is always higher than that appearing from a gap equation (which is a critical temperature for continuous phase transitions).

We also notice that the thermodynamic potential difference form can be easily applied to derive the critical magnetic field or magnetic induction, and we show that the heat capacity temperature dependence can be obtained directly from the order parameter vs. temperature plot. The latest interesting property should find simple experimental verification.

\section{Thermodynamic properties of $S$-paired system}

In order to consider the thermodynamic properties of any $S$-paired system in a weak coupling limit, we employ the standard formulas presented e.g. in [1-3]. The thermodynamic potential difference between the $S$-paired and the normal state can be written in the form [2,4]

$$
\Delta \Omega=N(0) T_{\mathrm{c}}^{2} \cdot \int_{0}^{Y}\left(\frac{\mathrm{d}}{\mathrm{d} Y^{\prime}} \frac{1}{\lambda}\right)\left(Y^{\prime}\right)^{2} \mathrm{~d} Y^{\prime},
$$

where $N(0)$ denotes the density of states per unit spin and volume on the Fermi surface and $\lambda=N(0) g$, a dimensionless coupling parameter is considered as a functional of the energy gap and temperature. The parameter $T_{\mathrm{c}}$, a constant in energy (temperature) scale, will be defined later, and

$$
Y=\frac{\Delta(T)}{T_{\mathrm{c}}}
$$

where $\Delta(T)$ is the energy gap dependent on temperature. Moreover, we introduce the following dimensionless symbols:

$$
X=\frac{T}{T_{\mathrm{c}}}, \quad \tau=\frac{Y}{2 X}=\frac{\Delta}{2 T} \quad \text { hence } \quad X=\frac{Y}{2 \tau} .
$$

In order to derive the thermodynamic potential difference $\left(\Delta \Omega=\Omega_{\mathrm{s}}-\Omega_{\mathrm{n}}\right)$ from Eq. (1) we should know the form of $\lambda$ as a function of $(Y, X)$ or $(Y, \tau)$. In spite of that, we can always assume that $1 / \lambda$ has the form

$$
\frac{1}{\lambda}=f(Y, \tau)
$$

where $f$ is an analytic function of $(Y, \tau)$. However, both variables

$$
Y=\frac{\Delta}{T_{\mathrm{c}}} \text { and } \tau=\frac{\Delta}{2 T}
$$

depend on $\Delta$ or equivalently on $\tau$.

Let us also note that the expression (4) defines the gap equation if $\lambda$ is assumed to be constant. Then putting

$$
\tau=0 \text { and hence } \Delta=0, T=T_{\mathrm{c}} \text { or } Y=0, X=1,
$$


or

$$
\tau=\infty \text { and hence } \Delta=\Delta_{0}, T=0 \text { or } Y=Y_{0}, X=0,
$$

we get

$$
\frac{1}{\lambda}=f(Y, \tau)=f\left(Y_{0}, \infty\right)=f(0,0)
$$

which allows us, after eliminating some other parameters, to derive $Y$ as a function of $X$, i.e. $\Delta=\Delta(T)$.

To execute more precise calculation we assume that the function $f(Y, \tau)$ can be separated to the form

$$
f(Y, \tau)=f_{0}(\tau)-F_{\infty} \ln Y,
$$

which is crucial for the considerations in the paper, where $f_{0}(\tau)$ is only a function of $\tau$ and $F_{\infty}$ is a constant, which turns out to be attainable in all considered cases $[1,2,5]$. We emphasize that the transformation of the function $f(Y, \tau)$ to the separated form Eq. (6) is possible only for the specifically chosen variables $Y$ and $\tau$.

Taking into account $f(Y, \tau)$ in the form of Eq. (6), we obtain from Eq. (1) (cf. Appendix)

$$
\frac{\Delta \Omega}{N(0) T_{\mathrm{c}}^{2} F_{\infty}}=-\frac{1}{2} Y^{2}(\tau)-8 X^{2}(\tau) \int_{0}^{\tau} \mathrm{d} \tau^{\prime} \tau^{\prime} \ln \frac{Y\left(\tau^{\prime}\right)}{Y(\tau)}
$$

which can be reduced to the form

$$
\frac{\Delta \Omega}{N(0) T_{\mathrm{c}}^{2} F_{\infty}}=-8 X^{2}(\tau) \int_{0}^{\tau} \mathrm{d} \tau^{\prime} \tau^{\prime} \ln \frac{X\left(\tau^{\prime}\right)}{X(\tau)}
$$

From Eqs. (7), (8) we can easy derive the entropy and the heat capacity differences between the $S$-paired and the normal state

$$
\frac{\Delta S}{16 N(0) T_{\mathrm{c}} F_{\infty}}=X(\tau) \int_{0}^{\tau} \mathrm{d} \tau^{\prime} \tau^{\prime} \ln \frac{Y\left(\tau^{\prime}\right)}{Y(\tau)}
$$

and

$$
\frac{\Delta C}{16 N(0) T_{\mathrm{c}} F_{\infty}}=\frac{\Delta S}{16 N(0) T_{\mathrm{c}} F_{\infty}}-\frac{1}{8} Y(\tau) \frac{\partial Y(\tau)}{\partial X(\tau)} .
$$

Let us discuss now the possibility of discontinuous phase transitions, which are owing to a finite leap of the order parameter $\Delta$. Equation (8) allows us to consider the problem for the quite general case, which can be formulated in the form of the following theorem.

Theorem

If there exists a parametric solution of the gap equation such that $\Delta$ and $T$ are the continuous functions of $\tau$, where $\tau=\Delta / 2 T$ is a parameter varying from zero $\left(\Delta=0, T=T_{\mathrm{c}}\right)$ to infinity $(\Delta=\Delta(0), T=0)$, and if non-zero (positive) values of $\Delta$ are achieved for $T>T_{\mathrm{c}}$ in every small vicinity of $T_{\mathrm{c}}$, then the order parameter is a discontinuous function of $T$ and it leaps to zero for $T_{\mathrm{c}}^{*}>T_{\mathrm{c}}(\tau=0)$. Proof

We employ the symbols introduced above. According to Eqs. (8) $\Delta \Omega$ is a continuous function of $\tau$, and $S$-paired state is realized if $\Delta(\Omega)=\Omega_{\mathrm{s}}-\Omega_{\mathrm{n}}<0$. 
Let $X(\tau)$ be an increasing function of $\tau$ for $0<\tau<\tau_{0}$ and $X(\tau)$ begins to decrease for $\tau>\tau_{0}$. For $\tau=\tau_{0}, X(\tau)$ reaches its maximum value. We can then distinguish an additional point $\tau_{1}>\tau_{0}$ for which $X\left(\tau_{1}\right)=1$. Moreover

$$
\begin{aligned}
& \text { if } 0<\tau<\tau_{1} \text { then } X(\tau)>1 \text {, } \\
& \text { if } \tau_{1}<\tau<\infty \text { then } X(\tau) \leq 1 \text { (and tends to zero). }
\end{aligned}
$$

Let us consider now the function $\Delta \Omega(\tau)$ for the specified values of $\tau$. Note that for $0<\tau \leq \tau_{0}$ we have

$$
\ln \frac{X\left(\tau^{\prime}\right)}{X(\tau)} \leq 0 \text { for } 0 \leq \tau^{\prime} \leq \tau \text { and } \Delta \Omega(\tau)>0
$$

On the other hand, if $\tau=\tau_{1}$ then

$$
\ln \frac{X\left(\tau^{\prime}\right)}{X\left(\tau_{1}\right)} \geq 0 \text { for } 0 \leq \tau^{\prime} \leq \tau_{1} \text { and } \Delta \Omega\left(\tau_{1}\right)<0 .
$$

Since $\Delta \Omega(\tau)$ is a continuous function of $\tau$ satisfying the conditions $\Delta \Omega\left(\tau_{0}\right)>0$ and $\Delta \Omega\left(\tau_{1}\right)<0$ there must exist a point $\tau_{2}$ such that $\tau_{0}<\tau_{2}<\tau_{1}$, for which $\Delta \Omega\left(\tau_{2}\right)=0$. Note that $X\left(\tau_{2}\right)>X\left(\tau_{1}\right)=1$, so the real transition temperature $T_{\mathrm{c}}^{*}$ is higher than $T_{\mathrm{c}}$ and can be defined by

$$
T_{\mathrm{c}}^{*}=T_{\mathrm{c}} X\left(\tau_{2}\right) \text {. }
$$

The derivation of $T_{\mathrm{c}}^{*}$ allows us to redefine all introduced magnitudes in respect to the real transition temperature, so we introduce the following symbols:

$$
X^{*}(\tau)=\frac{T}{T_{\mathrm{c}}^{*}}=X(\tau) \frac{T_{\mathrm{c}}}{T_{\mathrm{c}}^{*}}=\frac{X(\tau)}{X\left(\tau_{2}\right)} \quad \text { and } \quad Y^{*} s(\tau)=\frac{Y(\tau)}{X\left(\tau_{2}\right)} .
$$

Moreover, the order parameter (redefined energy gap) in the system under discussion, is a discontinuous function of temperature, and leaps from $\Delta\left(\tau_{2}\right)$ to zero at $T=T_{\mathrm{c}}^{*}$ (see Fig. 2a).

On the other hand, considering the parametric equations for the order parameter as functions of $\tau$, we have to assume that

$$
\text { for } 0 \leq \tau \leq \tau_{2} \quad X^{*}(\tau)=1 \text { and } Y^{*}(\tau)=2 \tau \text {. }
$$

For the new form of the order parameter, Eq. (8) reduces to the form

$$
\frac{\Delta \Omega}{N(0)\left(T_{c}^{*}\right)^{2} F_{\infty}}=-8\left[X^{*}(\tau)\right]^{2} \int_{\tau_{2}}^{\tau} \mathrm{d} \tau^{\prime} \tau^{\prime} \ln \frac{X^{*}\left(\tau^{\prime}\right)}{X^{*}(\tau)}+4 \tau_{2}^{2}\left[X^{*}(\tau)\right]^{2} \ln X^{*}(\tau) .
$$

Note that when $0 \leq \tau \leq \tau_{2}, \Delta \Omega(\tau)=0$. It enables us to to confine ourselves to the case of $\tau \geq \tau_{2}$, if we investigate the thermodynamic functions. Hence the entropy and the heat capacity difference (between the $S$-paired and the normal state), could be modified to the forms

$$
\frac{\Delta S(\tau)}{16 N(0) T_{c}^{*} F_{\infty}}=X^{*}(\tau) \int_{\tau_{2}}^{\tau} \mathrm{d} \tau^{\prime} \tau^{\prime}\left[\ln \frac{X^{*}\left(\tau^{\prime}\right)}{X^{*}(\tau)}-\frac{1}{2}\right]-\frac{1}{2} \tau_{2}^{2} X^{*}(\tau)\left[\ln X^{*}(\tau)+\frac{1}{2}\right]
$$

or equivalently

$$
\frac{\Delta S(\tau)}{16 N(0) T_{\mathrm{c}}^{*} F_{\infty}}=X^{*}(\tau) \int_{\tau_{2}}^{\tau} \mathrm{d} \tau^{\prime} \tau^{\prime} \ln \frac{Y^{*}\left(\tau^{\prime}\right)}{Y^{*}(\tau)}-\frac{1}{2} \tau_{2}^{2} X^{*}(\tau)\left(\ln X^{*}(\tau)+\ln \frac{\tau}{\tau_{2}}+\frac{1}{2}\right)
$$


and

$$
\frac{\Delta C(\tau)}{2 N(0) T_{c}^{*} F_{\infty}}=\frac{\Delta S(\tau)}{2 N(0) T_{c}^{*} F_{\infty}}-Y^{*}(\tau) \frac{\partial Y^{*}(\tau)}{\partial X^{*}(\tau)} .
$$

Note that if we put $\tau_{2}=0$, Eqs. (13), (15), (16) reduce to the forms of Eqs. (8), (9), (10), respectively. Moreover, taking into account the relation

$$
\Delta C=T \frac{\partial \Delta S}{\partial T}
$$

Eq. (16) becomes a nonhomogeneous linear differential equation. Solving it and using Eq. (17), after some calculations we can derive the entropy and the heat capacity difference in the forms

$$
\frac{\Delta S}{2 N(0) T_{c}^{*} F_{\infty}}=X \int_{X}^{1} \frac{Y^{2}\left(X^{\prime}\right)}{\left(X^{\prime}\right)^{3}} \mathrm{~d} X^{\prime}-\frac{1}{2} \frac{Y^{2}}{X}
$$

and

$$
\frac{\Delta C}{2 N(0) T_{\mathrm{c}}^{*} F_{\infty}}=X \int_{X}^{1} \frac{Y^{2}\left(X^{\prime}\right)}{\left(X^{\prime}\right)^{3}} \mathrm{~d} X^{\prime}-\frac{1}{2} \frac{Y^{2}}{X}-Y \frac{\partial Y}{\partial X},
$$

where we put $X=1$ for $T=T_{\mathrm{c}}$ or $T=T_{\mathrm{c}}^{*}$. The obtained forms of $\Delta S$ and $\Delta C$ are common for both cases under consideration. It proves that the heat capacity can be determined by the order parameter form (redefined energy gap) as a function of temperature.

Note that if $X \rightarrow 0$ we can assume that $Y$ is a slow-varying function of $X$ and hence we can easy estimate that $\Delta S$ and $\Delta C$ tend to zero. According to Eq. (18) the leap of the entropy at transition temperature can be written in the form

$$
\Delta S(X=1)=-N(0) T_{\mathrm{c}} F_{\infty}[Y(X=1)]^{2} .
$$

Similarly, we can define the leap of the heat capacity, employing Eq. (19), if we only can find the left-hand derivative of $\partial Y / \partial X$ in the limit $T \rightarrow T_{\mathrm{c}}$.

\subsection{Critical magnetic induction}

The critical magnetic field or the critical magnetic induction for $S$-paired systems can be easily found from the thermodynamic relations $[4,6]$. Therefore, applying Eq. (13), the critical magnetic induction can be presented in the form

$$
B_{\mathrm{c}}(T)=B_{\mathrm{c}}(0) \sqrt{\frac{\Delta \Omega}{\gamma_{0} N(0)\left(T_{\mathrm{c}}^{*}\right)^{2} F_{\infty}}},
$$

where

$$
B_{\mathrm{c}}(0)=T_{\mathrm{c}}^{*} \sqrt{2 \gamma_{0} \mu_{0} N(0) F_{\infty}}
$$

and

$$
\gamma_{0}=\frac{\Delta \Omega(\tau=\infty)}{N(0)\left(T_{c}^{*}\right)^{2} F_{\infty}}
$$

because the limit $\tau=\infty$ is equivalent to $T=0$. 


\section{Applications of the developed formalism}

In order to illustrate the power of the obtained results let us consider some particular $S$-paired Fermi systems.

\subsection{Statistical spin liquid}

Spałek et al. [7, 8] has generalized the standard Fermi-Dirac distribution for non-interacting electrons to deal with the statistical properties of strongly correlated electrons constituting, the so-called, statistical spin liquid. Czerwonko [5, 9] developed this model entirely discriminating the states of double spin occupancy.

We investigate thermodynamic properties of two specific systems, i.e. when the double spin occupancy of the same quasimomenta is forbidden and when the triple quasiparticle occupancy of the states with opposite quasimomenta is forbidden. Such distribution of quasiparticles causes that the spontaneous (unforced) creation of the Cooper pairs happens with the probability which is equal to $\frac{1}{2}$ and $\frac{1}{3}$, respectively. In order to derive thermodynamic functions for specified systems we employ the formalism established in $[1,2]$ and results achieved in $[5,9]$.

The basic element of the formalism, which allows us to determine properties of a system, is the kernel function of the gap equation. It can be taken in the form $[8]$

$$
F_{\propto}(\xi, \Delta(T))=\sum_{i=0}^{1} \frac{\sinh \left[\frac{1}{T} \sqrt{\xi^{2}+2 \Delta^{2}(T)}\right]}{2 \cosh \left[\frac{1}{T} \sqrt{\xi^{2}+2 \Delta^{2}(T)}\right]+4+\alpha \exp \left[(-1)^{i} \xi / T\right]},
$$

which causes that the gap equation can be written as follows (cf. Eq. (4) and [1]):

$$
\frac{1}{\lambda}=\int_{0}^{w_{\mathrm{D}}} \cdot \frac{F_{\alpha}(\xi, \Delta(T))}{\sqrt{\xi^{2}+2 \Delta^{2}(T)}} \mathrm{d} \xi
$$

where $\alpha=3$ and $\alpha=5$ for discussed above cases respectively, and $F_{\alpha}=$ $(2+\alpha) /[2(1+\alpha)]$ if $\xi \rightarrow \infty$.

Applying the established formalism and using the numerical methods, we can easily derive and analyse some characteristic properties of the $S$-paired statistical spin liquid. The obtained results are presented in the inserted plots. In Figs. 1a, 1b we present the forms of energy gaps and thermodynamic potential differences between $S$-paired and normal states (for $\alpha=3$ and $\alpha=5$ ), obtained from Eqs. (23) and (7), respectively. Since the results presented in Fig. 1a, 1b satisfy the conditions of the Theorem, we state that the phase transitions appear in fact in a critical temperature $T_{\mathrm{c}}^{*}>T_{\mathrm{c}}$ (see Fig. $2 \mathrm{a}, 2 \mathrm{~b}$ ). Its value can be derived from results presented in Fig. $2 \mathrm{~b}$ by defining the temperature for which $\Delta \Omega=0$.

According to developed formalism we must redefine the reduced temperature and introduce a discontinuous order parameter instead of the common used energy gap. Therefore, the energy gap is an order parameter only for phase transitions, where such order parameter is continuous.

In Figs. 2a, 2b we present the forms of the discontinuous order parameters and thermodynamic potential differences. The forms of the reduced entropy and heat capacity (or their differencies) as functions of reduced temperature are presented, in turn, in Figs. 3a-3d. They were obtained by means of Eqs. (15) and (16). 

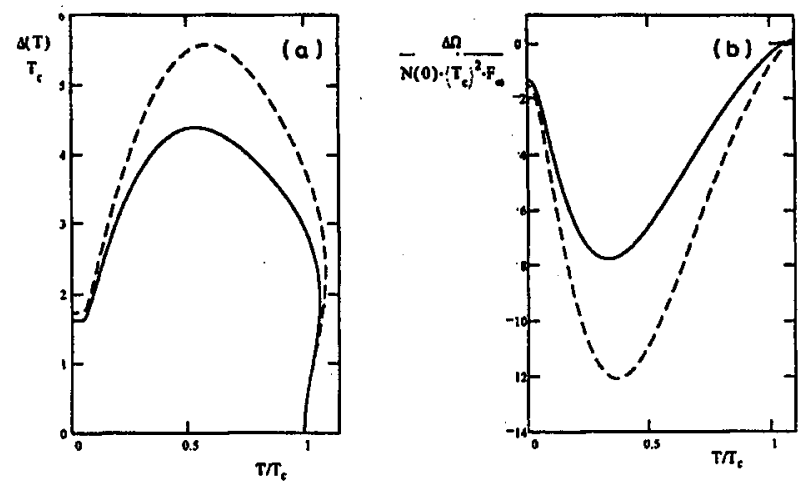

Fig. 1. (a) Energy gap $\Delta(T)$ as a function of reduced temperature $T / T_{\mathrm{c}}$ obtained for the two cases of statistical spin liquid model (see Eq. (22)), for $\alpha=3$ and $\alpha=5$ (dotted line). Coordinates of some characteristic and extreme points on the plots are given in a schematic form: for $\alpha=3-\left(T / T_{\mathrm{c}} ; \Delta(T) / T_{\mathrm{c}}\right):(0.00 ; 1.62),(0.53 ; 4.39),(1.91 ; 1.07)$; for $\alpha=5-\left(T / T_{\mathrm{c}} ; \Delta(T) / T_{\mathrm{c}}\right):(0.00 ; 1.73),(0.59 ; 5.58),(1.09 ; 2.33)$. (b) Thermodynamic potential difference $\Delta \Omega$ between the $S$-paired and normal state $\Delta \Omega=\Omega_{\mathrm{s}}-\Omega_{\mathrm{n}}$ as a function of reduced temperature for $\alpha=3$ and $\alpha=5$ (dotted line). Coordinates of some characteristic and extreme points on the plots are given in a schematic form: for $\alpha=3-\left(T / T_{\mathrm{c}} ; \Delta \Omega /\left[N(0) F_{\infty} T_{\mathrm{c}}^{2}\right]\right):(0.00 ;-1.32),(0.33 ;-7.77),(1.05 ; 0.00) ;$ for $\alpha=5-$ $\left(T / T_{c} ; \Delta \Omega /\left[N(0) F_{\infty} T_{c}^{2}\right]\right):(0.00 ;-1.52),(0.37 ;-12.08),(1.07 ; 0.00)$

We emphasize that the entropy of the normal statistical spin liquid is of the form

$$
S_{\mathrm{n}}=s_{0} N(0) \ln 2+\frac{2}{3} \pi^{2} N(0) T
$$

where $s_{0}$ is of order of characteristic energy $\epsilon_{\mathrm{F}}$, which ensures that the entropy of the normal and $S$-paired state is always positive, also at $T=0$ (cf. $[7,8]$ ).

The inserted curves show amazing properties of the $S$-paired statistical spin liquid model, developed by Czerwonko [5, 9]. The system reveals anomalous properties in the region of temperatures from 0 to 0.31 for $\alpha=3$ and to 0.34 for $\alpha=5$, since thermodynamic potential is a decreasing function of temperature (see Fig. 2b). Nevertheless, it could be connected with additional Cooper pairs creation. In this region, the increasing temperature enhances the pairing in the system of quasiparticles.

Moreover, as we can see in Fig. $3 \mathrm{~d}$, in the temperature region from 0.072 to $0.58(\alpha=3)$ and 0.078 to $0.61(\alpha=5)$, the heat capacity becomes negative, which is non-physical, and the system is unstable. We interpret this fact in this manner that since the number of Cooper pairs increases together with temperature, the thermodynamic potential decreases and the system emits an excessive energy i.e. heat, which additionally warms it.

In turn, in the temperature region above $0.58(\alpha=3)$ and $0.61(\alpha=5)$ the system shows a reasonable behaviour (see Fig. 3d).

For $T=T_{c}^{*}$ the heat capacity leaps to the value which corresponds to the normal phase, and height of the leap is equal to $4.50(\alpha=3)$ and $5.74(\alpha=5)$, 

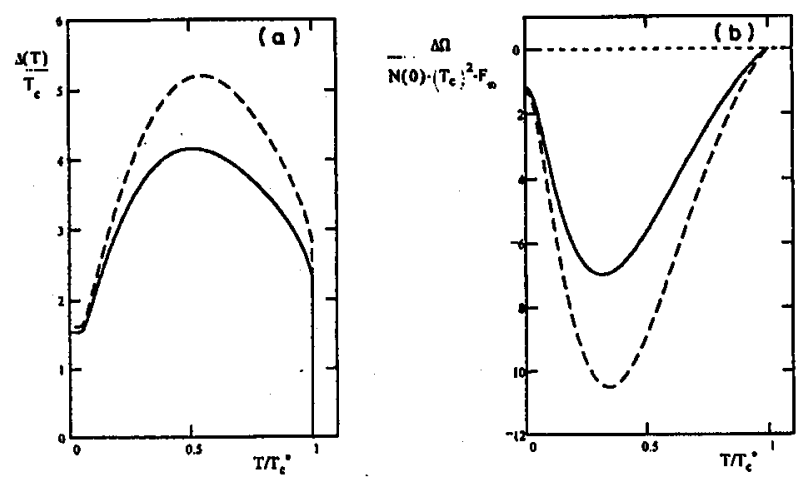

Fig. 2. (a) Discontinuous order parameter as a function of reduced temperature $T / T_{c}$ obtained for the two cases of statistical spin liquid model (see Eq. (22)), for $\alpha=3$ and $\alpha=5$ (dotted line) (see also Fig. 1a). Some characteristic and extreme points on the plots are given in a schematic form: for $\alpha=3-\left(T / T_{\mathrm{c}}^{*} ; \Delta(T) / T_{\mathrm{c}}^{*}\right)$ : $(0.00 ; 1.53),(0.51 ; 4.17),(1.00 ; 2.29)$; order parameter leaps rapidly from $\Delta\left(\tau_{2}\right)$ to 0 at $T=T_{c}^{*}$ for $\tau_{2}\left(=\Delta\left(T_{c}^{*}\right) / 2 T_{c}^{*}\right)=1.15 ;$ for $\alpha=5-\left(T / T_{c}^{*} ; \Delta(T) / T_{c}^{*}\right):(0.00 ; 1.61)$, $(0.55 ; 5.20),(1.00 ; 2.82)$; here order parameter leaps rapidly from $\Delta\left(\tau_{2}\right)$ to 0 at $T=T_{\mathrm{c}}^{*}$ for $\tau_{2}=1.41$. (b) Thermodynamic potential difference $\Delta \Omega$ between the $S$-paired and normal state $\Delta \Omega=\Omega_{\mathrm{s}}-\Omega_{\mathrm{n}}$ as a function of reduced temperature for $\alpha=3$ and $\alpha=5^{\alpha}$ (dotted line) (see also Fig. 1b). Characteristic and extreme points on the plots: for $\alpha=3-\left(T / T_{c}^{*} ; \Delta \Omega /\left[N(0) F_{\infty}\left(T_{c}^{*}\right)^{2}\right]\right):(0.00 ;-1.19),(0.31 ;-7.00) ;$ for $\alpha=5-$ $\left(T / T_{c}^{*} ; \Delta \Omega /\left[N(0) F_{\infty}\left(T_{c}^{*}\right)^{2}\right]\right):(0.00 ;-1.32),(0.34 ;-10.49)$.

respectively (see also Fig. 3d). We emphasize that the type of phase transition is determined by the order parameter behaviour. Since the order parameter vanishes abruptly at $T=T_{c}^{*}$ the phase transition of $S$-paired statistical spin liquid to the normal phase apparently must be of type $I$.

\subsection{Experimental data conversion}

The main results of the established formalism allow us to predict thermodynamic properties of the systems, defined in the previous section, basing only on the energy gap form. Such possibility seems to be very tempting in order to estimate some characteristic magnitudes taking use of experimental data.

However, to bring into practice one of two procedures of experimental data conversion given by Eqs. (7) (10) and (21) (method $A$ ) or Eqs. (18), (19) (method $B$ ) respectively, we have to define a reasonable differential curve being a function of the energy gap vs. temperature, which could be assumed as interpolation of experimental data.

The problem of fitting to experimental data is not very simple, even for a great number of experimental points, because always exists a wide choice of such curves due to the experimental data tolerance. Besides, functions encumber with errors under algebraic transformation, integration and differentiation bear finally larger errors. Nevertheless, being conscious of that, one should use a few fitted curves to evaluate the thermodynamic functions. 

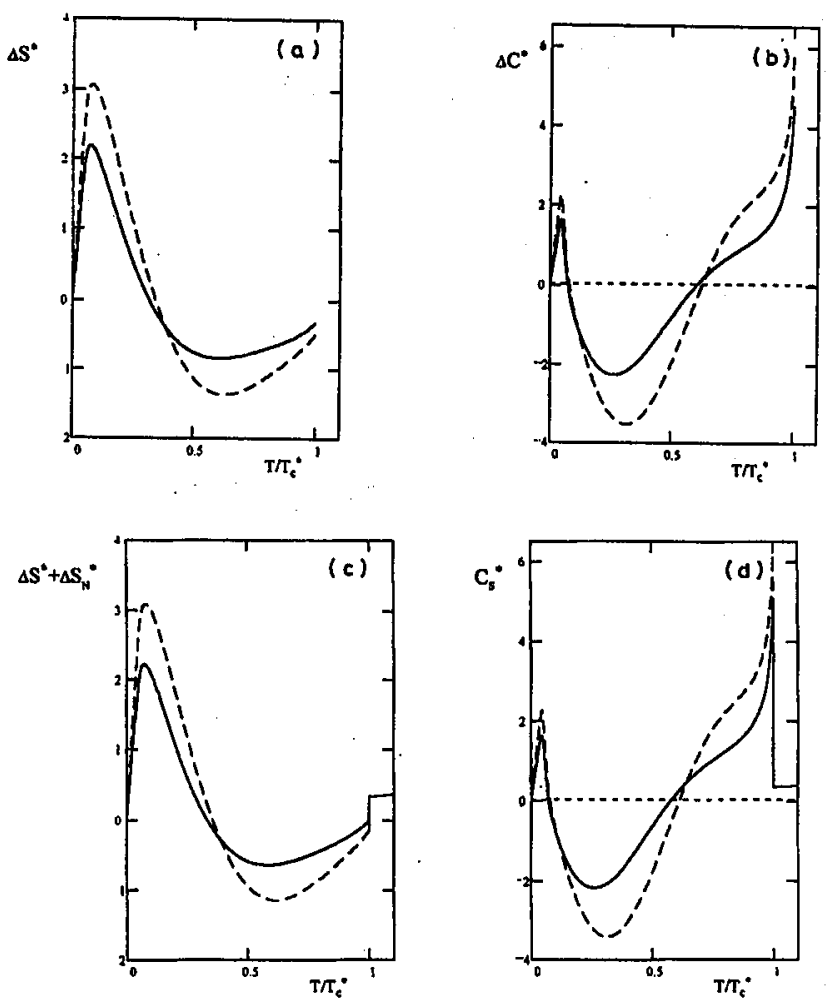

Fig. 3. (a) Reduced entropy as a function of reduced temperature for the two cases of statistical spin liquid model (see Eq. (22)), for $\alpha=3$ and $\alpha=5$ (dotted line). Coordinates of some characteristic and extreme points on the plots are given in a schematic form: for $\alpha=3-\left(T / T_{c}^{*} ; \Delta S^{*}\right):(0.07 ; 2.19),(0.61 ;-0.84),(1.00 ;-0.33) ;$ for $\alpha=5$ $-\left(T / T_{c}^{*} ; \Delta S^{*}\right):(0.08 ; 3.06),(0.63 ;-1.37),(1.00 ;-0.50)$; the maximum and minimum of $\Delta S^{*}$ must correspond to points where $\Delta C^{*}=0$ (see Fig. 3b). (b) Reduced heat capacity as a function of reduced temperature for $\alpha=3$ and $\alpha=5$ (dotted line). Characteristic and extreme points on the plots: for $\alpha=3-\left(T / T_{\mathrm{c}}^{*} ; \Delta C^{*}\right):(0.04 ; 1.60),(0.07 ; 0.00)$, $(0.26 ;-2.28) ;(0.61 ; 0.00),(1.00 ; 4.50) ;$ for $\alpha=5-\left(T / T_{c}^{*} ; \Delta C^{*}\right):(0.04 ; 2.21),(0.08 ; 0.00)$, $(0.31 ;-3.54) ;(0.63 ; 0.00),(1.00 ; 5.74)$. (c) See description of Fig. 3a. Extreme points on the plots: for $\alpha=3-\left(T / T_{c}^{*} ; \Delta S^{*}+\Delta S_{N}^{*}\right)$ : $(0.07 ; 2.22),(0.31 ; 0.10),(0.58 ;-0.65)$; $(1.00 ; 0.00)$; for $\alpha=5$ (dotted line) $-\left(T / T_{\mathrm{c}}^{*} ; \Delta S^{*}+\Delta S_{N}^{*}\right):(0.08 ; 3.08),(0.34 ; 0.12)$, $(0.61 ;-1.15) ;(1.00 ;-0.14)$; points where $C_{\mathrm{s}}=0$ correspond to extremes of $\Delta S^{*}+\Delta S_{N}^{*}$. (d) See description of Fig. 3b. Extreme points on the plots: for $\alpha=3-\left(T / T_{c}^{*} ; C_{s}^{*}\right)$ : $(0.04 ; 1.60),(0.07 ; 0.00),(0.26 ;-2.20) ;(0.58 ; 0.00)$ where $C_{s}^{*}$ leaps at $T=T_{c}^{*}$ from 4.83 to 0.33 ; for $\alpha=5$ (dotted line) $-\left(T / T_{\mathrm{c}}^{*} ; C_{\mathrm{s}}^{*}\right):(0.04 ; 2.23),(0.08 ; 0.00),(0.31 ;-3.43)$, $(0.61 ; 0.00)$; here $C_{\mathrm{s}}^{*}$ leaps at $T=T_{\mathrm{c}}^{*}$ from 6.09 to 0.35 .

In Figs. 4a-4e and Figs. 5a-5c we present, according to method $A$ and $B$ respectively, some results obtained for experimental data of the energy gap given in the paper [10]. The investigations which we have carried out allow us to notice 

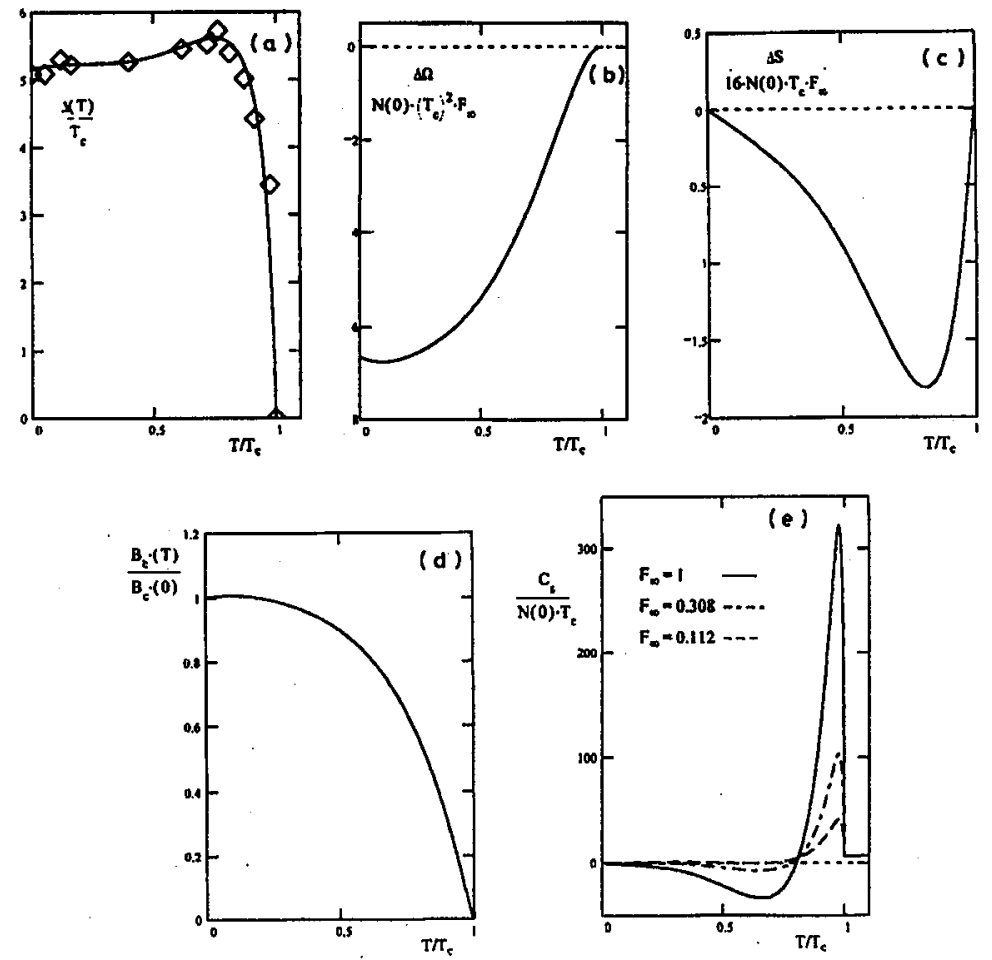

Fig. 4. (a) Interpolated curve of the energy gap $\Delta(T)$ as a function of reduced temperature for high- $T_{c}$ superconductor, basing on experimental data from tunneling spectroscopy (from the paper [10]). (b) Thermodynamic potential difference $\Delta \Omega=\Omega_{\mathrm{s}}-\Omega_{\mathrm{n}}$ vs. reduced temperature obtained basing on the interpolated curve of the energy gap from Fig. 4a. The extreme points of the plot: $\left(T / T^{*}{ }_{c} ; \Delta \Omega /\left[N(0) F_{\infty} T_{c}^{2}\right]\right):(0.00 ;-6.67)$, $(0.20 ;-6.77)$ (the minimum). (c) Reduced entropy difference $\Delta S=S_{\mathrm{s}}-S_{\mathrm{n}}$ vs. reduced temperature obtained basing on the interpolated curve of the energy gap from Fig. $4 \mathrm{a}$. The minimum of the plot: $\left(T / T_{\mathrm{c}} ; \Delta S /\left[16 N(0) F_{\infty} T_{\mathrm{c}}^{2}\right]\right):(0.81 ;-1.81)$. (d) Reduced critical magnetic induction vs. reduced temperature obtained basing on the interpolated curve of the energy gap from Fig. 4a. The maximum of the plot: $\left(T / T_{\mathrm{c}} ; B_{\mathrm{c}}(T) / B_{\mathrm{c}}(0)\right)$ : $(0.10 ; 1.007)$. (e) Reduced heat capacity $C_{\mathrm{s}}$ of the superconducting phase vs. reduced temperature obtained basing on the interpolated curve of the energy gap from Fig. 4a. Characteristic points on the plots: for $\left(F_{\infty}=1.00\right)-\left(T / T_{\mathrm{c}} ; C_{\mathrm{s}} /\left[N(0) F_{\infty} T_{\mathrm{c}}\right]\right):(0.66 ;-33.20)$, $(0.80 ; 0.00),(0.98 ; 319) ;$ for $\left(F_{\infty}=0.308\right)-\left(T / T_{\mathrm{c}} ; C_{\mathrm{s}} /\left[N(0) F_{\infty} T_{\mathrm{c}}\right]\right):(0.65 ;-7.27)$, $(0.78 ; 0.00),(0.98 ; 104) ;$ for $\left(F_{\infty}=0.112\right)-\left(T / T_{c} ; C_{\mathrm{s}} /\left[N(0) F_{\infty} T_{\mathrm{c}}\right]\right):(0.32 ; 1.07)$, $(0.6 \mathbb{Z} ; 0.00),(0.98 ; 42.20)$. All parts a-e are obtained by method $A$.

a few general properties of the energy gap, entropy and heat capacity differences, which always occur in all extended BCS or $S$-paired systems, for which crucial Eq. (6) is fulfilled. Moreover, if the experimental data prove that the tested superconductor does not possess such properties, it means that its theoretical model cannot be based on extended BCS regime. 

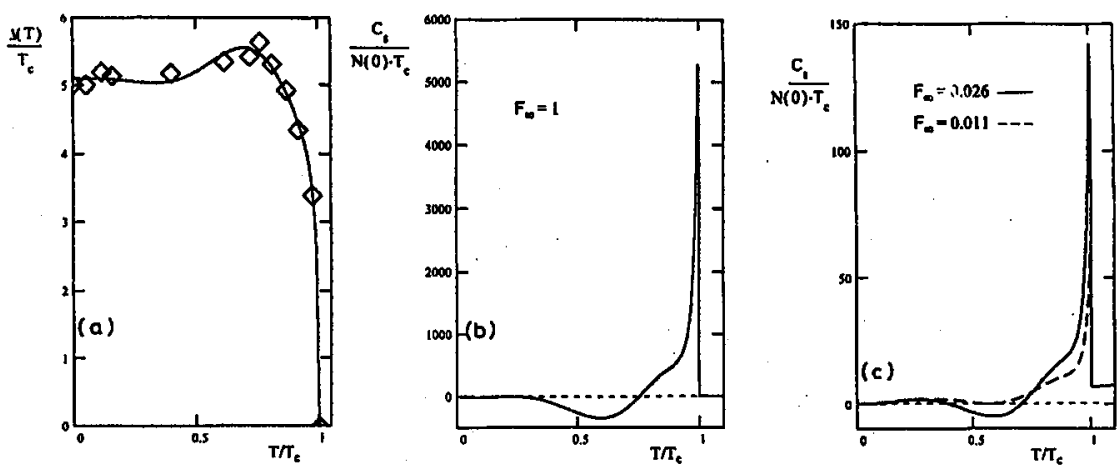

Fig. 5. (a) Slightly different, comparing to Fig. 4a, form of interpolated curve of the energy gap, basing on experimental data of HTSC's tunneling spectroscopy (taken from the paper [10]) (see also description of Fig. 4a). (b), (c) Reduced heat capacities $C_{\mathrm{s}}$ of the superconducting phase vs. reduced temperature obtained basing on the new interpolated curve of the energy gap from Fig. 5a. Characteristic points on the plots for $F_{\infty}=0.011, F_{\infty}=0.026$ and $F_{\infty}=1.00$ are the following: $\left(T / T_{c} ; C_{s} /\left[N(0) F_{\infty} T_{c}\right]\right)$ : $(0.31 ; 1.77),(0.56 ; 0.00),(1.00 ; 63.80) ;\left(T / T_{\mathrm{c}} ; C_{\mathrm{s}} /\left[N(0) F_{\infty} T_{c}\right]\right):(0.27 ; 1.56),(0.39 ; 0.00)$, $(0.58 ;-5.19),(0.70 ; 0.00),(1.00 ; 142) ;\left(T / T_{\mathrm{c}} ; C_{\mathrm{s}} /\left[N(0) F_{\infty} T_{c}\right]\right):(0.59 ;-348.00),(0.75 ; 0.00)$, $(1.00 ; 5250)$. In Fig. $5 \mathrm{a} \Delta(0) / T_{\mathrm{c}}=5.1$. All parts a-c are obtained by method $B$.

Equation (19) allows us to derive the heat capacity difference curve vs. temperature based on a fitted curve of the energy gap (Figs. 5b, 5c). According to the third law of thermodynamics the heat capacity has to tend to zero if temperature tends to zero. Note that for the normal state

$$
C_{\mathrm{n}}=\frac{2}{3} \pi^{2} N(0) T_{\mathrm{c}} X
$$

and hence $\Delta C=C_{\mathrm{s}}-C_{\mathrm{n}}$ must tend to zero in the limit $T \rightarrow 0$. Investigating Eq. (19) we state that the above condition is always fulfilled if the energy gap satisfies the relation

$$
\left(\frac{\partial \Delta T}{\partial T}\right)_{T=0}=\left(\frac{\partial Y(X)}{\partial X}\right)_{X=0}=0
$$

Thus, the last Eq. (26) constitutes the new fundamental condition which confines the class of extended BCS-type superconductors. Let us rewrite Eq. (19) in the form

$$
\frac{\Delta C}{16 N(0) T_{\mathrm{c}} F_{\infty}}=X \int_{X}^{1} \frac{Y^{2}\left(X^{\prime}\right)-Y^{2}(X)}{\left(X^{\prime}\right)^{3}} \mathrm{~d} X^{\prime}-\frac{1}{2} X Y^{2}(X)-Y(X) \frac{\partial Y(X)}{\partial X} .
$$

The precise analysis of Eq. (27) proves that in the limit $X \rightarrow 0 \Delta C$ will contain two main terms proportional to $X$ and to $\partial Y(X) / \partial X$, respectively

$$
\Delta C=-a X-b \frac{\partial Y(X)}{\partial X}
$$

where, in general, we cannot fix the sign of $a$ and $b$. 
In the standard BCS model, realized in low temperature superconductors, $a=\frac{2}{3} \pi^{2} N(0) T_{\mathrm{c}}$, and hence $C_{s} \sim \partial Y(X) / \partial X$. However, in more complicated systems like high- $T_{\mathrm{c}}$ superconductors, those two constants do not need to be identical or $a$ can be neglected, then $C_{s} \sim X$ since $\partial Y(X) / \partial X<X$.

On the other hand, the established formalism contains one additional parameter $F_{\infty}$, suitable choice of which, allows us to eliminate the linear term from $C_{\mathbf{s}}$ (see Eq. (28)). In practice, to compute the values of thermodynamic functions, $F_{\infty}$ should be known. Note that according to Eqs. (18) and (19) the similar properties as $\Delta C$ possess also $\Delta S$ i.e. $\Delta S$ tends to zero if $T \rightarrow 0$.

Let us consider now the properties of $\Delta C$ if temperature tends to its critical value. Examining Eq. (27) we can state that the first and the second terms of $\Delta C$ become small if $T \rightarrow T_{\mathrm{c}}$ for continuous phase transitions. Thus, the shape of the heat capacity difference curve depends only on the expression $Y(X) \partial Y(X) / \partial X$. Since if $X \rightarrow 1$ then $Y \rightarrow 0$ and the $\Delta C$ converges to a non-zero value only if $-\partial Y(X) / \partial X$ tends to infinity. It means that if the slope of the energy gap at $T=T_{\mathrm{c}}$ is finished $(-\partial Y(X) / \partial X<\infty, X=1)$, the heat capacity difference achieves zero. Note that $\Delta S$ achieves zero at $T=T_{\mathrm{c}}$ always, for continuous phase transitions.

In Figs. $4 a-4 \mathrm{e}$ we present results of our experimental data conversion, i.e. thermodynamic potential and entropy differences (Figs. 4b, 4c), heat capacity (Fig. 4e) and critical magnetic induction (Fig. 4d) of the real superconductor [10], obtained according to Eqs. (7)-(10) and (21), which corresponds to method $A$ in the paper. Plots are presented for different values of parameter $F_{\infty}$ (Fig. 4e).

Each of the above discussed conditions allow us to fix the value of the parameter $F_{\infty}$ independently. However, if the $C_{\mathrm{s}}$ tends to zero in non-linear way, like in BCS model, there exists a temperature interval, in which the $C_{\mathrm{s}}$ achieves negative values. But on the other hand, if we require $C_{\mathrm{s}} \geq 0$ for all temperatures, then $C_{\mathbf{s}}$ tends linearly to zero with temperature.

Concluding we emphasize that the local maximum in the energy gap implies the local minimum in the heat capacity of the superconductor, and in consequence it may take negative values.

\section{Conclusions}

Though the developed formalism has the phenomenological character, it was obtained basing on microscopic Green functions approach for $S$-paired Fermi systems (e.g. [11]). The presented parametric forms of thermodynamic functions admit the possibility of existence of discontinuous order parameter (leap the energy gap to zero). Due to the fast convergent integrals, parametric formulas are very convenient for numerical calculations. It allows us to make a quick verification of results obtained in some microscopic approaches.

Besides, the advantage of the formalism expresses also in fact that we can derive the thermodynamic functions basing only on the experimental data of energy gap (see Sec. 3.2).

Moreover, since the form of the energy gap determines the thermodynamic functions, its form can be reconstructed from them. 
The work was supported in part by the Committee for Scientific Research grant No. PB 2 P302 12506.

\section{Appendix}

Below we present precise calculations which allow us to obtain the general form of the thermodynamic potential differences between the $S$-paired and the normal state

$$
\begin{aligned}
& \frac{\Delta \Omega}{N(0)\left(T_{\mathrm{c}}\right)^{2}}=\int_{0}^{Y}\left[\frac{\mathrm{d}}{\mathrm{d} Y^{\prime}} \frac{1}{\lambda}\right]\left(Y^{\prime}\right)^{2} \mathrm{~d} Y^{\prime}=\int_{0}^{Y}\left[\frac{\mathrm{d}}{\mathrm{d} Y^{\prime}}\left(Y^{\prime}, \tau^{\prime}\right)\right]\left(Y^{\prime}\right)^{2} \mathrm{~d} Y^{\prime} \\
& =\int_{0}^{Y} \frac{\mathrm{d}}{\mathrm{d} Y^{\prime}}\left[f_{0}\left(\tau^{\prime}\right)-F_{\infty} \ln Y^{\prime}\right]\left(Y^{\prime}\right)^{2} \mathrm{~d} Y^{\prime} \\
& =\int_{0}^{Y}\left[\frac{\partial f_{0}}{\partial \tau^{\prime}} \frac{\mathrm{d} \tau^{\prime}}{\mathrm{d} Y^{\prime}}-F_{\infty} \frac{\partial \ln Y^{\prime}}{\partial Y^{\prime}}\right]\left(Y^{\prime}\right)^{2} \mathrm{~d} Y^{\prime} \\
& =\int_{0}^{Y} \frac{\partial f_{0}}{\partial \tau^{\prime}}\left(Y^{\prime}\right)^{2} \frac{\mathrm{d} \tau^{\prime}}{\mathrm{d} Y^{\prime}} \mathrm{d} Y^{\prime}-F_{\infty} \int_{0}^{Y} Y^{\prime} \mathrm{d} Y^{\prime} \\
& =-\frac{1}{2} F_{\infty} Y^{2}+4 X^{2} f_{0} \tau^{2}-8 X^{2} \int_{0}^{\tau} f_{0} \tau^{\prime} \mathrm{d} \tau^{\prime},
\end{aligned}
$$

where we replaced $Y$ by $2 X \tau$. Now, if we employ the relation

$$
f_{0}(\tau)=\frac{1}{\lambda}+F_{\infty} \ln Y(\tau)
$$

and hence we get

$$
\begin{gathered}
\frac{\Delta \Omega}{N(0)\left(T_{\mathrm{c}}\right)^{2}}=-\frac{1}{2} F_{\infty} Y^{2}+4 X^{2} \tau^{2}\left[\frac{1}{\lambda}+F_{\infty} \ln Y(\tau)\right] \\
-8 X^{2} \int_{0}^{\tau}\left[\frac{1}{\lambda}+F_{\infty} \ln Y\left(\tau^{\prime}\right)\right] \tau^{\prime} \mathrm{d} \tau^{\prime}=F_{\infty}\left[-\frac{1}{2} Y^{2}-8 X^{2} \int_{0}^{\tau} \ln \frac{Y\left(\tau^{\prime}\right)}{Y(\tau)} \tau^{\prime} \mathrm{d} \tau^{\prime}\right] .
\end{gathered}
$$

\section{References}

[1] R. Gonczarek, M. Mulak, Acta Phys. Pol. A 86, 1007 (1994).

[2] M. Mulak, R. Gonczarek, Acta Phys. Pol. A 89, 689 (1996).

[3] M. Mulak, R. Gonczarek, Czech. J. Phys. 46, 3379 (1996).

[4] A.A. Abrikosov, Fundamental Theory of Metals, North-Holland, Amsterdam 1988.

[5] J. Czerwonko, Physica $C$ 235-240, 2307 (1994).

[6] A.L. Fetter, J.D. Walecka, Kwantowa teoria uktadów wielu czastek, PWN, Warszawa 1988 (in Polish).

[7] J. Spałek, W. Wójcik, Phys. Rev. B 37, 1532 (1988).

[8] J. Spałek, Phys. Rev. B 40, 5180 (1989).

[9] J. Czerwonko, Mol. Phys. Rep. 12, 79 (1995).

[10] H. Enomoto, H. Kokado, I. Matsubara, N. Mori, H. Ozaki, Czech. J. Phys. 46, 1331 (1996).

[11] R. Gonczarek, Acta Phys. Pol. A 71, 59 (1986). 